\title{
A stochastic differential equation approach to modelling the growth phase of fire spread
}

\author{
$\underline{\text { S. Sanni }}^{\text {a }}$, J. J. Sharples ${ }^{\text {a }}$, A. M. Gill ${ }^{\text {b }}$, Z. Jovanoski ${ }^{\text {a }}$, H. S. Sidhu ${ }^{\text {a }}$, and I. N. Towers ${ }^{\text {a }}$ \\ ${ }^{a}$ School of Physical, Environmental and Mathematical Sciences, UNSW Canberra, ACT 2600 \\ ${ }^{b}$ Fenner School of the Environment and Society, ANU, ACT 0200 \\ Email: s.sanni@student.adfa.edu.au
}

\begin{abstract}
From a point source, landscape fires accelerate until they reach a quasi-equilibrium rate of spread. The rate at which a fire accelerates from its ignition affects the time first responders have to attack a fire in its initial stages when it is more easily suppressed. As such, knowledge of the rate of acceleration of a fire from ignition can be valuable from a fire management perspective. However, the majority of studies in wildland fire science have been dedicated to development of models for the quasi-equilibrium rate of spread attained by the fire after its acceleration phase. Comparatively little attention has been given to the development of models that specifically account for the growth phase of a fires development.
\end{abstract}

The rate of acceleration depends on many factors including variations in ambient and induced wind speed and direction, variation in moisture content of the fuel, fuel stratification and slope variation. Present models of fire growth from a point ignition are expressed as deterministic algebraic equations, thereby neglecting variability. The numerous variables involved make predictions of rate of spread from a point source very difficult.

In this paper we consider two approaches to model the acceleration phase of a fire. The first considers fitting a sigmoidal function to experimental data using a nonlinear curve-fitting procedure. The model takes the following functional form:

$$
R(t)=\begin{gathered}
R_{0} R_{e} \\
\left(R_{0}+\left(R_{e}-R_{0}\right) \exp (-a t)\right)
\end{gathered}
$$

where $R(t)$ is the rate of spread at any time $t$. The parameters $R_{0}$ and $R_{e}$ are the initial and quasi-equilibrium rate of spread respectively, and $a$ is the growth rate.

In the second approach we propose the use of stochastic differential equations to investigate the growth of a fire to quasi-equilibrium. Specifically, the model is given by the following stochastic differential equation:

$$
d R=a R\left(1-\frac{R}{R_{e}}\right) d t+\sigma R d W
$$

subject to the initial condition $R(0)=R_{0}$. Here, $W$ is a Wiener process.

In addition to providing a more realistic portrayal of the time series data relating to fire growth, this second approach allows for better discrimination of the mechanisms driving the growth phase of fire spread.

The models are assessed by appealing to observations of experimental fire g rowth. Specifically the data relate to fires growing from a point ignition under the influence of a uniform wi nd. The re sults indicate that both approaches can provide an accurate representation of the observed data, but that the approach based on stochastic differential equations yields $95 \%$ confidence bounds that are higher than those obtained from the nonlinear curve-fitting. The difference in confidence bounds indicates that the way stochasticity is incorporated into fire growth models has implications for how models inform decisions about the likelihood of a fire self-extinguishing before it reaches quasi-equilibrium, and the magnitude of the rates of spread it is likely to exhibit during the initial stages of growth.

Keywords: Fire growth, fire acceleration, rate of spread, stochastic processes, stochastic differential equations 


\section{INTRODUCTION}

Bushfires are a significant environmental problem. In the last decade this has become increasingly apparent, with a number of major conflagrations causing extensive loss of life and property, and considerable environmental damage. Regardless of the ultimate size and intensity of a bushfire, all fires start small - often originating as a point ignition, or something akin to a point ignition, and growing in a manner that is governed by the ambient environmental conditions. In addition, under certain conditions a bushfire will deposit firebrands ahead of the main front, igniting spot-fires, which can also be viewed as evolving from a point source.

Landscape fires originating from a point source accelerate until they reach a quasi-equilibrium rate of spread. This behaviour has also been confirmed in laboratory experiments (McAlpine \& Wakimoto 1991). Attacking fires while they are in their acceleration phase, before they have attained their potential quasi-steady rate of spread, provides a greater chance of suppression success. As such, there are good reasons to understand the likely behaviour of a fire during its acceleration phase, and mathematical modelling provides a means to improve this understanding. However, in comparison with the vast literature that deals with modelling the quasi-equilibrium rate of spread, there is little that specifically considers this acceleration phase of a fires development (Cruz et al. 2015). The manner in which a fire develops during its acceleration phase depends on the local environmental conditions, including variations in ambient and induced wind speed and direction, and variations in moisture content and structure of the fuel. Even when a fire attains a quasi-equilibrium state, its rate of spread can still exhibit considerable variation about a mean value.

However, present models of fire growth from ignition are expressed as deterministic algebraic equations, thereby neglecting variability. Parameters in such models are estimated using nonlinear regression of empirical data, and once determined they are considered as fixed; separate estimates are required for different fuel types and environmental conditions. Any resulting model lacks flexibility and should not be used in circumstances where the fuel and environmental conditions are heterogeneous.

Moreover, interactions between winds, terrain, different vegetation types and structures means that the conditions affecting the initial growth of a fire (and its longer term propagation) can never be adequately quantified in solely deterministic terms. Coupling between the fire and the atmosphere further confound this situation. The intrinsic variability of the factors affecting the initial growth of a fire means that deterministic modelling approaches are ill-suited to modelling this aspect of fire spread. Indeed, such approaches gloss over the detailed dynamics, which are inherently stochastic in nature due to the turbulent conditions under which fires develop. Recent advances in stochastic dynamical systems theory and applications, and in our understanding of dynamic fire behaviour mean that we are now in a position to explicitly address the acceleration phase (from a point ignition) of fire growth in terms of stochastic dynamic systems.

The models we propose in this paper appear to be the first to consider the acceleration phase of a fire's growth as a stochastic dynamical system. Such an approach permits a probabilistic interpretation of fire growth, which allows for estimation of worst-case and mean-case scenarios. Also, by explicitly accounting for the intrinsic variability of fire spread and the environmental conditions that drive it, our stochastic approach has the potential to provide greater insights into the physical processes governing fire growth, particularly in its acceleration phase.

\section{DATA AND METHODS}

\subsection{Rate of spread data}

The rate of spread (Ros) data used in this study are taken from McAlpine \& Wakimoto (1991). The data were extracted using G3DATA (Frantz 2000) and are reproduced in Figure 1. The data set represents five replicates of the Ros observed in a wind tunnel experiment. In each case the fuel load was $26.3 \mathrm{~kg} / \mathrm{m}^{3}$ and burned under the influence of a wind speed of $4.8 \mathrm{~km} /$ hour. The temperature $\left(26.7^{\circ} \mathrm{C}\right)$ and humidity $(80 \%)$ were controlled for. The fuel moisture content was measured just prior to burning, and the mean of the five replicates was $8.62 \%$ (standard deviation of 0.57 ). It is of fundamental interest that even with carefully controlled conditions and a fixed wind speed, the variation in Ros can be substantial. As already mentioned, this variation may be due to differing fuel moisture content, variability in the packing of the fuel bed for each experimental run (Finney 2004), and turbulence induced by the fire itself. We treat each of the replicates in Figure 1 as independent data sets. 


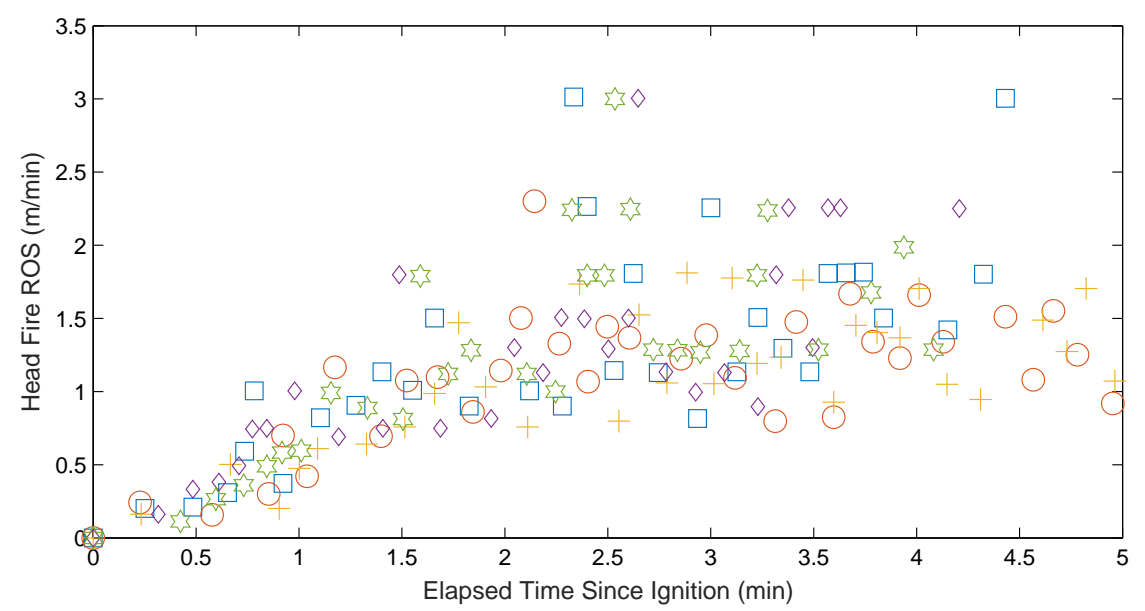

Figure 1. Observed rate of spread for a pine needle fuel bed, with a bulk density of $26.3 \mathrm{~kg} / \mathrm{m}^{3}$ and burned at a wind speed of $4.8 \mathrm{~km} / \mathrm{hr}$. Five replications are shown, each symbol (square, circle, etc.) being a separate replication. (Figure recreated from McAlpine \& Wakimoto (1991).)

\subsection{Nonlinear curve-fitting}

Typically a model for the Ros, $R(t)$, is obtained by postulating a functional form with a set of unknown parameters that need to be determined. The shape of the functional form is driven by the data. McAlpine \& Wakimoto (1991) provide a summary of some well known models that are relevant for different environmental conditions and fuel types. These include exponential-type models such as $R(t)=R_{e} \exp (-\alpha / t)$ and $R(t)=$ $R_{e}(1-\exp (-\alpha t))$, and power-law models, $R(t)=\beta t^{\alpha}$. In all cases $\alpha, \beta$ and $R_{e}$ are constants that need to be determined. In both exponential models $R(t) \rightarrow R_{e}$ as $t \rightarrow \infty$, where $R_{e}$ is the asymptotic or quasiequilibrium Ros, whereas the power-law model never attains a quasi-equilibrium state.

Taking into account the data in Figure 1, it appears that there is an initial rapid increase in the Ros until reaching an inflection point, after which the acceleration begins to decrease, asymptotically approaching the quasi-equilibrium Ros. To describe this behaviour, we propose a sigmoid function:

$$
R\left(t_{i}\right)=\frac{R_{0} R_{e}}{\left(R_{0}+\left(R_{e}-R_{0}\right) \exp \left(-a t_{i}\right)\right)}+\epsilon_{i}, \quad i=1,2, \ldots, n,
$$

for $n$ data points $\left(t_{i}, R\left(t_{i}\right)\right)$. Here $R_{0}$ is the initial value of the Ros, the quasi-equilibrium value is $R_{e}$ and $a$ is the rate of increase of the Ros. The $\epsilon_{i}$ 's are independent and identically distributed errors. The basic assumptions of regression analysis are assumed except constant variance. The latter is justified since the variability in the data tends to increase with increasing Ros.

The aim is to estimate the three parameters, $R_{0}, R_{e}$ and $a$, by implementing a weighted nonlinear least-squares method to minimise the sum of the weighted square of the errors (or weighted residuals) between the observed $R\left(t_{i}\right)$ and the curve-fitted values for each $t_{i}$. The weighted sum of squares is

$$
L_{w}\left(R_{0}, R_{e}, a\right)=\sum_{i=1}^{n} w_{i}\left(R\left(t_{i}\right)-\frac{R_{0} R_{e}}{R_{0}+\left(R_{e}-R_{0}\right) \exp \left(-a t_{i}\right)}\right)^{2}
$$

where $w_{i}$ are the weights. The weights determine how much each $R\left(t_{i}\right)$ value influences the final parameter estimates. Here the residuals are weighted using a bi-square weighting scheme. This method minimises a weighted sum of squares, where the weight given to each data point depends on how far the point is from the fitted line. Points near the line get full weight. Points farther from the line get reduced weight. Points that are farther from the line than would be expected by random chance get zero weight. The bi-square weights are given by

$$
w_{i}= \begin{cases}\left(1-\left(u_{i}\right)^{2}\right)^{2}, & \left|u_{i}\right|<1 \\ 0, & \left|u_{i}\right| \geq 1\end{cases}
$$


where the $u_{i}$ are the standardised adjusted residuals. Robust fitting with bi-square weights uses an iteratively re-weighted least-squares algorithm.

The minimisation of $L_{w}$ with respect to the parameters must be carried out iteratively. The LevenbergMarquardt method is a standard technique for solving nonlinear least squares problems (Rowlings et al. 1998). The Levenberg-Marquardt curve-fitting method is a combination of two minimisation methods: the gradient descent method and the Gauss Newton method. In the gradient descent method, the sum of the squared errors is reduced by updating the parameters in the steepest-descent direction. In the Gauss-Newton method, the sum of the squared errors is reduced by assuming that the least squares function is locally quadratic, and finding the minimum of the quadratic. The Levenberg-Marquardt method acts more like a gradient-descent method when the parameters are far from their optimal value, and acts more like the Gauss-Newton method when the parameters are close to their optimal value. Both options are available in MATLAB.

\subsection{A stochastic differential equation approach}

An alternative to curve-fitting is to reformulate the model as a differential equation (DE), specifically as a stochastic differential equation (SDE). For a dynamic process such as the spread of a fire it seems more natural to employ a DE or an SDE. Using an SDE allows for the inclusion of local environmental variability that might be due to heterogeneous fuels or changing wind direction and strength. With reference to Figure 1, during the early development of the fire, $R^{\prime}(t) \propto R(t)$, then as $R(t) \rightarrow R_{e}, R^{\prime}(t) \rightarrow 0$. This behaviour is adequately captured by the logistic DE. The stochastic version of the logistic DE written in the Ito form is (Gardiner 2009),

$$
d R=a R\left(1-\frac{R}{R_{e}}\right) d t+\sigma R d W, \quad R(0)=R_{0},
$$

where the parameters $R_{0}, R_{e}$ and $a$ have the same meaning as before. The first term on the right hand side is the drift and the second term is the stochastic term, often called the diffusion. The parameter $\sigma$ is the strength of the local environmental variability and $W(t)$ is a standard Wiener process with expected value $\mathbb{E}[W(t)]=0$ and variance $\mathbb{V}[W(t)]=t$. The appearance of $R(t)$ in the stochastic term assumes that the variance increases with increase in the Ros. For $\sigma=0$ the solution to (2) is the sigmoid function (1). This provides a basis for comparing the two modelling approaches.

\section{RESULTS AND DISCUSSION}

For illustrative purposes we fit the curve expressed through (1) to a single replicate of the data (the squares) in Figure 1 . The null hypothesis is that both $R_{e}$ and $a$ are zero, compared to the alternate hypothesis that at least one of $R_{e}$ and $a$ is non-zero. The estimated parameters including a 95\% confidence interval (CI) is shown in Table 1:

Table 1. Nonlinear curve-fitting to a single replicate (square symbol)

\begin{tabular}{|c|c|c|c|c|c|}
\hline Parameter & $R_{0}$ & $R_{e}$ & $a$ & RMSE & $\mathrm{R}^{2}$ \\
\hline Value & 0.203 & 1.576 & 1.622 & 0.445 & 0.648 \\
\hline $95 \% \mathrm{CI}$ & $(-0.109,0.516)$ & $(1.248,1.904)$ & $(0.135,3.109)$ & & \\
\hline
\end{tabular}

According to the estimated values and their CIs there is statistical evidence to reject the null hypothesis. The Root Mean Square Error (RMSE) is the standard deviation of the weighted residuals - a measure of spread of the difference between the predicted and observed values. The estimated $\mathrm{R}^{2}$ indicates that about $65 \%$ of the variability in the data is accounted for by the fitted curve. Furthermore, plots of weighted residuals indicate homoscedasticity of the variance and a lack of correlation. The fitted model provides a good fit to the data ( see Figure refreal).

The solution to (2) typically requires a numerical approach, the simplest of these is the Euler-Maruyama algorithm (Higham 2001). At the heart of this algorithm is the following discretisation of the SDE

$$
R_{i+1}=R_{i}+a R_{i}\left(1-\frac{R_{i}}{R_{e}}\right) \Delta t_{i}+\sigma R_{i} \Delta W_{i}
$$


where $\Delta t_{i}=t_{i+1}-t_{i}, \Delta W_{i}=W_{i+1}-W_{i}=\sqrt{\Delta t_{i}} \xi_{i}$ and $\xi_{i} \sim N(0,1)$. The numerical solution to the SDE is a single realisation, but what is of importance is to determine the expected value and variance of a large number of such realisations.
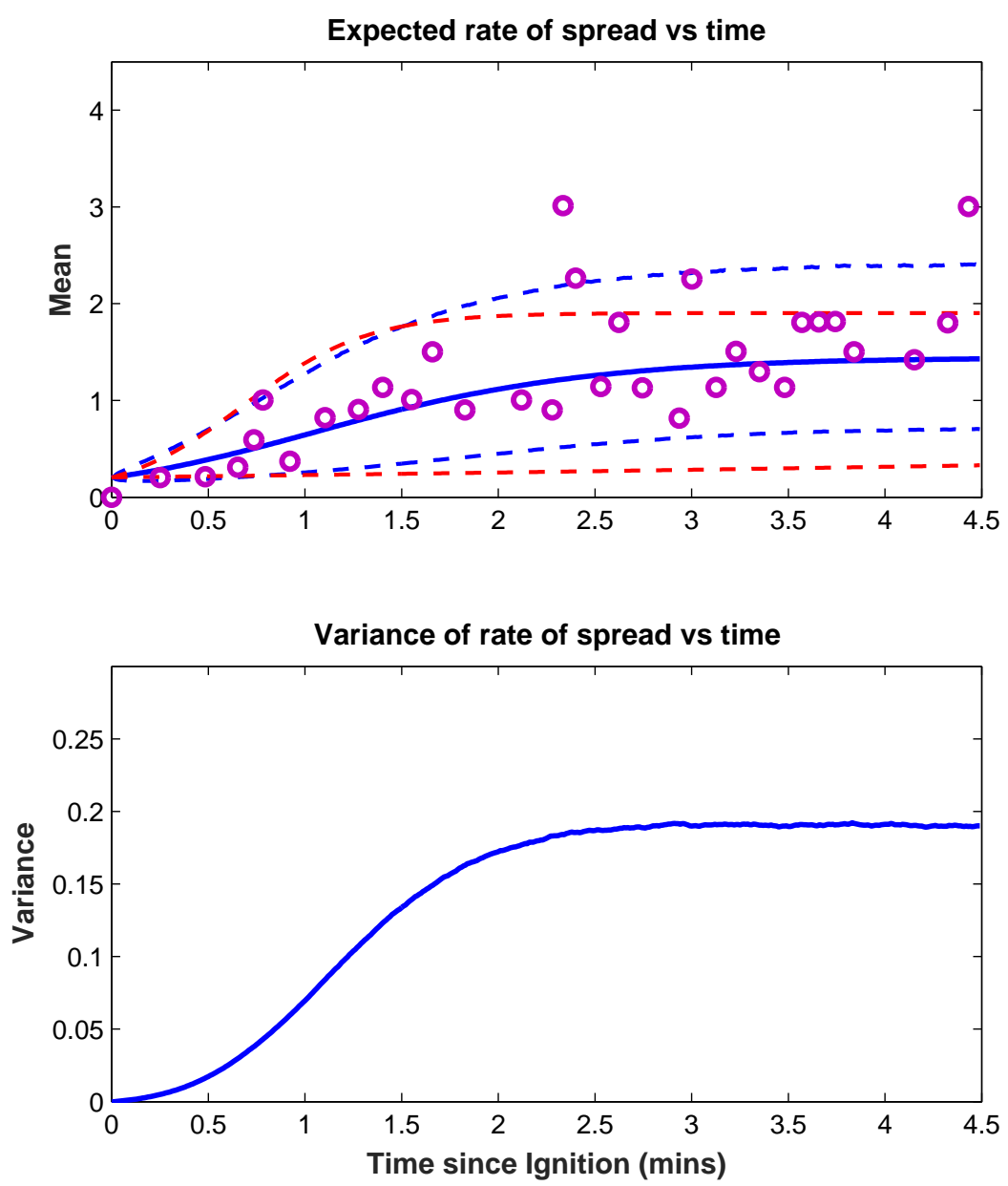

Figure 2. Comparison of the nonlinear curve-fitting and simulations based on the SDE. The top panel shows the $95 \%$ CIs for the fitted curve (red dashed line) and that of the SDE (blue dashed line). The mean Ros using both approaches is the same and is given by the solid line. The data is represented by circles. The bottom panel shows the simulated variance in the Ros based on the Monte Carlo simulation of (3). Here $\Delta t=0.01$.

To solve equation (2) we must specify four parameters: $R_{0}, R_{e}, a$ and $\sigma$. Strictly speaking we should estimate these parameters by using maximum-likelihood methods or other related techniques directly to (3) (Kloeden et al. 1994). Here, however, we make use of the values already determined previously, and our tasks remains to find $\sigma$. This is done by searching through a range of values of $\sigma$ for which the estimated variance $\mathbb{V}[R(t)]$ (estimated at $t \approx 4.5 \mathrm{~min}$ ) is equal to the MSE (square of the RMSE). This is justifiable if we seek to make a comparison between (1) and (3).

To estimate both $\mathbb{E}[R(t)]$ and variance $\mathbb{V}[R(t)]$ we employ an Monte Carlo approach with 100,000 realisations. Figure 2 depicts the results of the 100,000 realisations. The top panel show the expected Ros with a $95 \% \mathrm{CI}$, where as the bottom panel shows the variance of the Ros. Requiring $\mathbb{V}[R(t)] \approx$ MsE yields $\sigma \approx 0.72$. It is clear that almost all of the data resides within the $95 \% \mathrm{CI}$, suggesting that the proposed model provides a good description for the observed Ros. 
Over the time domain relevant for the duration of the experiment, the lower of the $95 \%$ CI of the fitted curve offers practical information about the likelihood of the fire self-extinguishing, often referred to as a 'go/nogo' threshold. The closer the lower $\mathrm{CI}_{\mathrm{I}}$ is to zero, the more likely the fire will self-extinguish. The upper CI provides important information for fire management, as it relates to the potential for rapid initial growth of a fire in the landscape. Knowledge of the upper CI can therefore give fire managers a better idea of the operational window for initial response once an ignition occurs, thus enabling more informed deployment of personnel and assets to head off a fire during its initial stages.

It is of interest to note that both of the CIs for the SDE model are higher than those of the fitted curve. Since they both have the same variance, the difference must be intrinsic to the way stochasticity is accounted for in each model. The results imply that a fire whose behaviour is more in accord with the SDE model is less likely to self-extinguish, and has the potential to exhibit higher rates of spread.

Computing a large number of realisations as in Figure 2 allows us to generate statistics concerning fire spread. Figure 3 shows a frequency distribution of the Ros at four different times: $1 \mathrm{~min}, 2 \mathrm{~min}, 3 \mathrm{~min}$ and $4 \mathrm{~min}$. Superimposed is a continuous curve obtained via kernel smoothing to help aid in identifying the location of the distribution and the skewness that is apparent at earlier times. The distribution is narrow at earlier times, the distribution continues to evolve and by $t=3 \mathrm{~min}$ the frequency distribution remains relatively unchanged, having achieved a quasi-equilibrium state. From this we can estimate the probability that the Ros is larger than a particular value, or lies within some range of values, at any time $t$ after ignition.
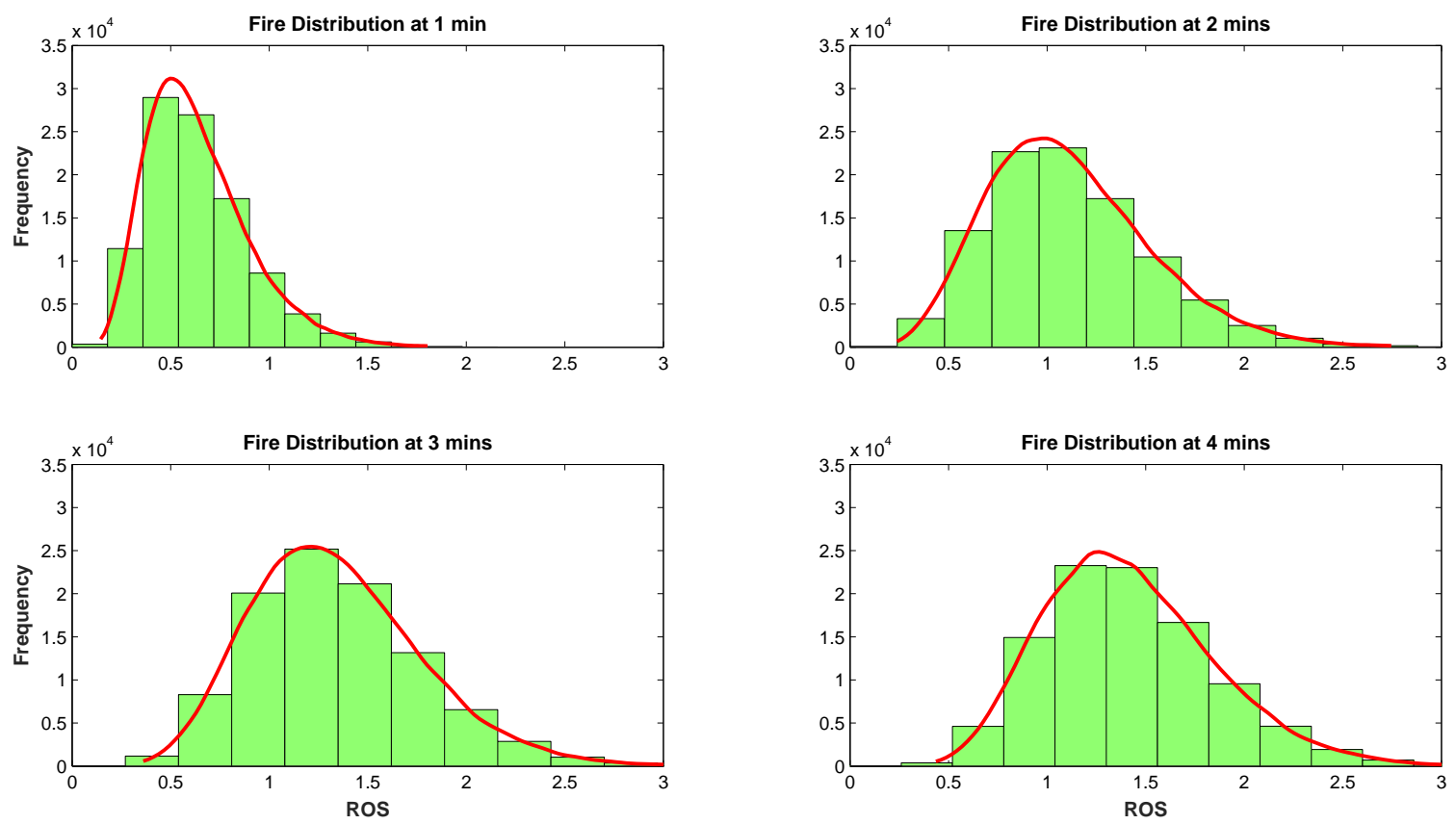

Figure 3. The evolution of the frequency distribution for the Ros based on 100,000 realisations of (3).

The proposed model is a first step to modelling the fire growth from ignition by using an SDE. The manner by which stochasticity is added is a modelling issue that deserves further inquiry. Equation (2) incorporates stochasticity by aggregating the effect of growth and self limiting, both of which are affected by local environmental variability, into a single term.

More generally, if $\mathbf{x}$ represents a set of (possibly variable) conditions including fuel type, wind direction and strength, and even factors such as fire front curvature (Weber 1989), then each member of the set can also be realised as the solution of an SDE coupled to (2) through the state-dependent parameters $a(\mathbf{x})$ and $R_{e}(\mathbf{x})$ (Gardiner 2009). This provides a way of incorporating the effects of environmental variability during the 
acceleration phase of fire spread across a landscape.

\section{CONCLUSIONS}

In developing models for the spread of fire it is customary to fit the data based on some prescribed functional form. Such methods provide good descriptions of the time evolution of the mean ROS from time of igni-tion. The aim is to obtain parameter estimates and relevant CIs for the spread rate. Alternatively, an SDE formulation is a natural way to describe the rate of change of the ROS, a quantity that can be experimentally measured. Carrying out a large number of simulations enables the construction of an empirical probability density function for the ROS, from which statistical quantities can be determined. We found that once the ROS reaches quasi-equilibrium the upper bound of the CI is larger in the SDE formulation than that determined by curve-fitting. This has practical implications in the management of fires.

The model we propose explicitly incorporates the stochastic dynamics of fire spread and environmental vari-ability. Through our model we seek to provide a more appropriate method for assessing the likely development of fires in the landscape. It is expected that the model can be used to better inform the decisions that need to be made by fire managers through provision of a probabilistic framework that acknowledges the uncertainties inherent in fire spread.

The SDE formulation for the spread rate is particularly attractive as it provides a way to explicitly involve a fires history (Viegas 2004). This could be accomplished by using either a distributed timedelay in the spread rate, which involves a weighted average of the spread rate taken over all previous times, or as a discrete time-lag due to the process of pyrogenesis (Dold \& Zionviev 2009). This line of research is currently being investigated.

\section{ACKNOWLEDGEMENT}

S. Sanni gratefully acknowledges the financial support of a Faculty Research Grant (UNSW Canberra).

\section{REFERENCES}

Cruz, M. G., Gould, J. S., Alexander, M. E., Sullivan, A. L., McCaw, W. L. \& Matthews, S. (2015). A guide to rate of fire spread models for Australian vegetation, Technical report, CSIRO Land and Water Flagship, Canberra, ACT, and AFAC, Melbourne, Vic, 123pp.

Dold, J. W. \& Zionviev, A. (2009). 'Fire eruption through intensity and spread-rate interaction mediated by flow attachment', Combustion Theory and Modelling 13, 763-793.

Finney, M. A. (2004). Farsite: Fire area simulator - model development and evaluation, Technical report, USDA For. Serv., Rocky Mt. Res. Stn., Ogden, UT. Res. Pap. RMRS-RP-4 Revised. 47 p.

Frantz, J. (2000). 'G3DATA', http://www.frantz.fi/software/g3data.php. [Online: accessed March 20, 2011 ].

Gardiner, C. (2009). Stochastic Methods: A handbook for the natural and social sciences, Springer-Verlag, Berlin.

Higham, D. J. (2001). 'An algorithmic introduction to numerical simulation of stochastic differential equations', SIAM. Review 43, 525-546.

Kloeden, P. E., Platen, E. \& Schurz, H. (1994). Numerical solution of SDE through computer experiments, Springer-Verlag, Berlin.

McAlpine, R. S. \& Wakimoto, R. H. (1991). 'The acceleration of fire from point source to equilibrium spread', Forest Science 37, 1314-1337.

Rowlings, J. O., Pantula, S. G. \& Dickey, D. A. (1998). Applied regression analysis: a research tool, 2 edn, Springer-Verlag, New York.

Viegas, D. X. (2004). 'On the existence of a steady state regime for slope and wind driven fires', International Journal of Wildland Fire 13, 101-117.

Weber, R. O. (1989). 'Analytic models for fire spread due to radiation', Combustion and Flame 78, 398-408. 